\title{
trt-1 Is the Caenorhabditis elegans Catalytic Subunit of Telomerase
}

\author{
Bettina Meier ${ }^{1,2 \times a}$, luval Clejan ${ }^{1,3}$, Yan Liu ${ }^{1 \times b}$, Mia Lowden ${ }^{1,4}$, Anton Gartner ${ }^{2}$, Jonathan Hodgkin ${ }^{5}$, Shawn Ahmed ${ }^{1,3,4^{*}}$ \\ 1 Department of Genetics, University of North Carolina, Chapel Hill, North Carolina, United States of America, 2 Wellcome Trust Biocenter, University of Dundee, Dundee, \\ United Kingdom, 3 Lineberger Comprehensive Cancer Center, University of North Carolina, Chapel Hill, North Carolina, United States of America, 4 Department of Biology, \\ University of North Carolina, Chapel Hill, North Carolina, United States of America, 5 Genetics Unit, Department of Biochemistry, University of Oxford, Oxford, United \\ Kingdom
}

Mutants of trt-1, the Caenorhabditis elegans telomerase reverse transcriptase, reproduce normally for several generations but eventually become sterile as a consequence of telomere erosion and end-to-end chromosome fusions. Telomere erosion and uncapping do not cause an increase in apoptosis in the germlines of trt-1 mutants. Instead, lategeneration trt-1 mutants display chromosome segregation defects that are likely to be the direct cause of sterility. trt-1 functions in the same telomere replication pathway as mrt-2, a component of the Rad9/Rad1/Hus1 (9-1-1) proliferating cell nuclear antigen-like sliding clamp. Thus, the 9-1-1 complex may be required for telomerase to act at chromosome ends in C. elegans. Although telomere erosion limits replicative life span in human somatic cells, neither trt-1 nor telomere shortening affects postmitotic aging in $C$. elegans. These findings illustrate effects of telomere dysfunction in C. elegans mutants lacking the catalytic subunit of telomerase, trt-1.

Citation: Meier B, Clejan I, Liu Y, Lowden M, Gartner A, et al. (2006) trt-1 is the Caenorhabditis elegans catalytic subunit of telomerase. PLoS Genet 2(2): e18.

\section{Introduction}

Telomeres in most organisms are composed of simple repetitive sequences whose length is maintained by the telomerase ribonucleoprotein, which reverse transcribes telomere repeat sequences onto chromosome ends. Two subunits of telomerase are sufficient for generating telomerase activity in vitro: the telomerase reverse transcriptase (TERT) and the telomerase RNA, which contains the template sequence used by TERT for telomere repeat addition [1-3]. In the absence of telomerase, the DNA replication machinery is unable to completely replicate the chromosome terminus and telomere attrition occurs [4].

Most normal human somatic cells are deficient for TERT and therefore experience progressive telomere shortening when proliferating $[1,2,5]$. Telomere erosion in human primary cells grown in vitro typically causes replicative senescence within 40 to 60 population doublings [6]. Telomere erosion is exacerbated in patients with Werner's progeria (a segmental aging syndrome), and the in vitro premature senescence phenotype of Werner's cells can be overcome by expression of TERT [7]. Further, shortened life span and decreased cellular proliferative capacity are observed for late-generation mouse telomerase RNA mutants [8], and mice deficient for both the telomerase RNA and the Werner helicase display a variety of premature aging phenotypes in only a few generations $[9,10]$. These results provide compelling evidence that telomere erosion may affect proliferative aging in humans.

The nematode Caenorhabditis elegans was selected to study the impact of telomerase and telomere length in an organismal context. C. elegans has telomeres composed of simple TTAGGC repeats that are 2 to 4 kilobases in length [11]. The first C. elegans telomere replication mutant to be identified, mortal germline-2 (mrt-2), was recovered in a genetic screen for mortal germline mutants that are initially fertile but become progressively sterile if passaged for multiple generations [12]. mrt-2 mutants become sterile as a consequence of progressive telomere shortening and endto-end chromosome fusions. In addition, mrt-2 mutants are hypersensitive to ionizing radiation, and $m r t-2$ encodes an ortholog of a subunit of the 9-1-1 proliferating cell nuclear antigen (PCNA)-like sliding clamp that is loaded onto aberrant DNA structures at sites of genome damage [12]. Further evidence that the 9-1-1 DNA damage response complex is fully required for telomere replication in C. elegans is provided by HUS-1, another subunit of the 9-1-1 PCNA-like heterotrimer that physically interacts with MRT-2 [13]. Mutation of hus-1 also results in telomere erosion, end-to-end chromosome fusions, and late-onset sterility [14]. Here we report the identification of the $C$. elegans catalytic subunit of telomerase, trt-1, and several consequences of telomere dysfunction in strains deficient for $t r t-1$.

Editor: Stuart Kim, Stanford University, United States of America

Received September 2, 2005; Accepted January 3, 2006; Published February 10, 2006

A previous version of this article appeared as an Early Online Release on January 3, 2006 (DOI: 10.1371/journal.pgen.0020018.eor)

DOI: 10.1371/journal.pgen.0020018

This is an open-access article distributed under the terms of the Creative Commons Public Domain declaration which stipulates that, once placed in the public domain, this work may be freely reproduced, distributed, transmitted, modified, built upon, or otherwise used by anyone for any lawful purpose.

Abbreviations: bp, base pair; DAPI, 4',6-diamidino-2-phenylindole; PCNA, proliferating cell nuclear antigen; TERT, telomerase reverse transcriptase

* To whom correspondence should be addressed. E-mail: shawn@med.unc.edu aa Current address: School of Life Sciences, University of Dundee, Dundee, United Kingdom

ab Current address: Laboratory of Biochemistry and Genetics, National Institute of Diabetes and Digestive and Kidney Diseases, National Institutes of Health, Bethesda, Maryland, United States of America 


\section{Synopsis}

In the 1930s, the maize geneticist Barbara McClintock observed that broken chromosome ends occasionally became stable when transmitted through the germline, and she inferred that a protective cap present at normal chromosome ends could be added to broken chromosomes de novo. We now know that short, repetitive DNA sequences cap chromosome ends in most eukaryotes and that such sequences can be added de novo by the telomerase reverse transcriptase, which uses an RNA template for telomere repeat addition. The authors genetically define the reverse transcriptase subunit of telomerase in the roundworm Caenorhabditis elegans. Proteins that respond to abnormal DNA damage are required for telomere replication in C. elegans, suggesting that chromosome ends may be recognized as a special form of DNA damage prior to telomere repeat addition by telomerase. The authors found that telomerase and DNA damage response proteins appear to function in the same telomere replication pathway. Replicative aging in human somatic cells may be caused by telomere shortening. However, somatic cells do not proliferate in C. elegans adults, and telomere erosion does not affect the aging process in this context. Finally, the authors observed that chromosome mis-segregation may explain the progressive sterility of C. elegans telomerase mutants.

\section{Results}

\section{Identification of the C. elegans Telomerase Reverse Transcriptase}

Based on previous observations with mrt-2 and hus-1 [12,14], continuous propagation of $C$. elegans telomere replication mutants was expected to result in uncapping and fusion of de-protected chromosome ends as a consequence of telomere erosion. C. elegans end-to-end chromosome fusions produce a dominant chromosome loss phenotype when heterozygous [12]. A small pilot screen of 200 ethylmethane sulfonatemutagenized F2 lines yielded a single mortal germline mutation, $e 2727$, that displayed a dominant Him (high incidence of males or X-chromosome nondisjunction) phenotype when late-generation $e 2727$ hermaphrodites were crossed with N2 wild-type males. Genetic mapping showed tight linkage of the dominant Him phenotype to one end of the $\mathrm{X}$ chromosome and to an end of an autosome in each case (Figure 1A), indicating the presence of $\mathrm{X}$-autosome end-to-end chromosome fusions. These chromosome fusions were viable when homozygous, suggesting that their breakpoints were close to chromosome termini (unpublished data). Further, microscopy of late-generation $e 2727$ hermaphrodites revealed fewer than the normal complement of six bivalents in oocytes, the only C. elegans cells that arrest in metaphase and are therefore amenable to quantification of chromosome number, confirming the presence of end-to-end chromosome fusions (Figure 1B). Southern analysis of telomere length in $e 2727$ indicated that this mutant displayed progressive telomere shortening (Figure S1). The Mortal Germline phenotype of $e 2727$ was used to map this mutation to a genetic location of +3 on Chromosome I (six of nine Uncnon-Dpy recombinants from $+e 2727+$ I unc- $13+d p y-24$ F1 heterozygotes carried $e 2727$ and unpublished data). BLAST analysis identified a nearby gene, DY3.4, with weak but significant homology to telomerase reverse transcriptases. Sequencing of DY3.4 in the $e 2727$ mutant revealed a spliceacceptor mutation for intron 5 (Figure 1C), which resulted in mis-splicing and nonsense-mediated degradation of $90 \%$ of the DY3.4 message in $e 2727$ (unpublished data), as judged by RT-PCR analysis in a $s m g-2$ background, which is defective for nonsense-mediated mRNA decay [15]. However, $10 \%$ of the DY3.4 message is still spliced correctly in $s m g-2, e 2727$ doubles, as commonly occurs for C. elegans splice-junction mutations.

A second allele of DY3.4, yp 1, was identified in a large-scale screen for mortal germline mutants as a telomere replication mutant that mapped to the center of Chromosome I (Y. Liu and S. Ahmed, unpublished data). Two additional alleles of DY3.4, ok410 and tm899, were recovered by groups searching for deletions of DY3.4 in pools of mutagenized worms. Consistent with the $e 2727$ mutant phenotypes, $y p 1$, ok410, and tm899 strains initially had normal brood sizes but displayed late-onset sterility over successive generations. A reduced chromosome complement was observed for late-generation strains near sterility (Figure 1B) but not for early or middle generations (unpublished data). Further, a dominant Him phenotype was observed for $\mathrm{F} 1$ derived from late-generation $y p 1, o k 410$, and $t m 899$ hermaphrodites crossed with wild-type males (unpublished data). Genetic mapping of the dominant Him phenotype from a number of independent $o k 410$ strains revealed tight linkage to one end of the $\mathrm{X}$ chromosome and to the end of an autosome in each case (M. Lowden and S. Ahmed, unpublished data), confirming that end-to-end chromosome fusions had been isolated. As $y p 1$, ok410, and tm899 appeared to have telomere replication defects caused by mutations in or near the DY3.4 gene, complementation tests were conducted. The $e 2727, y p 1$, and $t m 899$ mutations all failed to complement $o k 410$, but not a tightly linked mutation in unc-29, for the late-onset sterility phenotype (unpublished data), indicating that $e 2727, y p 1, t m 899$, and $o k 410$ are allelic.

The DY3.4 gene was sequenced in $y p 1$ and found to contain a G-to-A splice acceptor mutation for intron 6, which results in retention of this intron and generation of a premature stop codon at the intron-exon boundary. The DY3.4 deletions ok410 and tm899 eliminate coding sequence for several essential reverse transcriptase motifs of the DY3.4 protein and therefore ought to be null alleles (Figure 1C). Southern blotting of genomic DNA prepared from trt-1 mutants revealed comparable rates of telomere erosion for $0 k 410$ (125 \pm 17.5 base pairs [bp]/generation), $\operatorname{tm} 899$ (128.8 \pm 27.5 bp/generation), yp1 (111.3 $\pm 18.3 \mathrm{bp} /$ generation), and $e 2727$ (123.6 $\pm 16.5 \mathrm{bp} /$ generation) (Figures $2 \mathrm{~A}$ and $\mathrm{S} 1$ ), despite the fact that $e 2727$ expressed some correctly spliced DY3.4 messenger RNA. The Mortal Germline phenotypes of $e 2727$ and $o k 410$ were rescued by extrachromosomal arrays carrying the wild-type DY3.4 gene, as nonrescued siblings of two $e 2727$ and one ok410 strain became sterile within nine or ten generations postinjection, whereas their rescued siblings were still fertile after 32, 22, and 17 generations, respectively. Rescue of ok410 was transient, probably as a consequence of progressive silencing of the rescuing transgene, as is commonly observed for transgenes in the C. elegans germline. Analysis of telomere length by Southern blotting revealed that the telomere shortening phenotypes of $e 2727$ and $o k 410$ were also rescued by the wild-type DY3.4 gene, which conferred the diffuse telomeres expected from stochastic telomere repeat addition by telomerase (Figure 2B and unpublished data). Moreover, staining of oocyte nuclei with 4',6-diamidino-2-phenylindole (DAPI) revealed that only about $1 \%$ of $e 2727$ and $o k 410$ hermaphrodites carrying a rescuing array for ten generations contained chromosome 
A

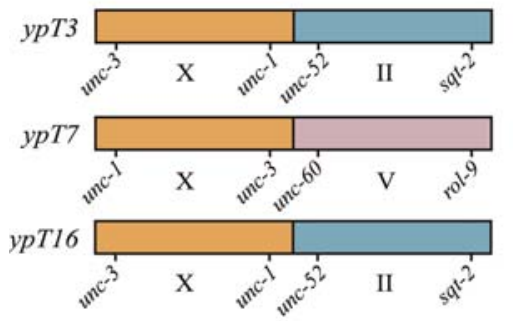

B

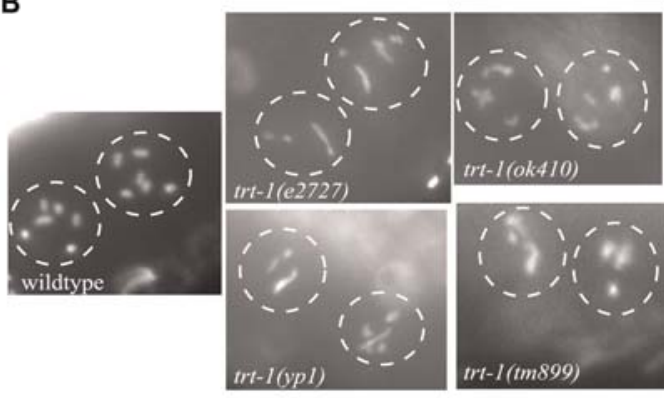

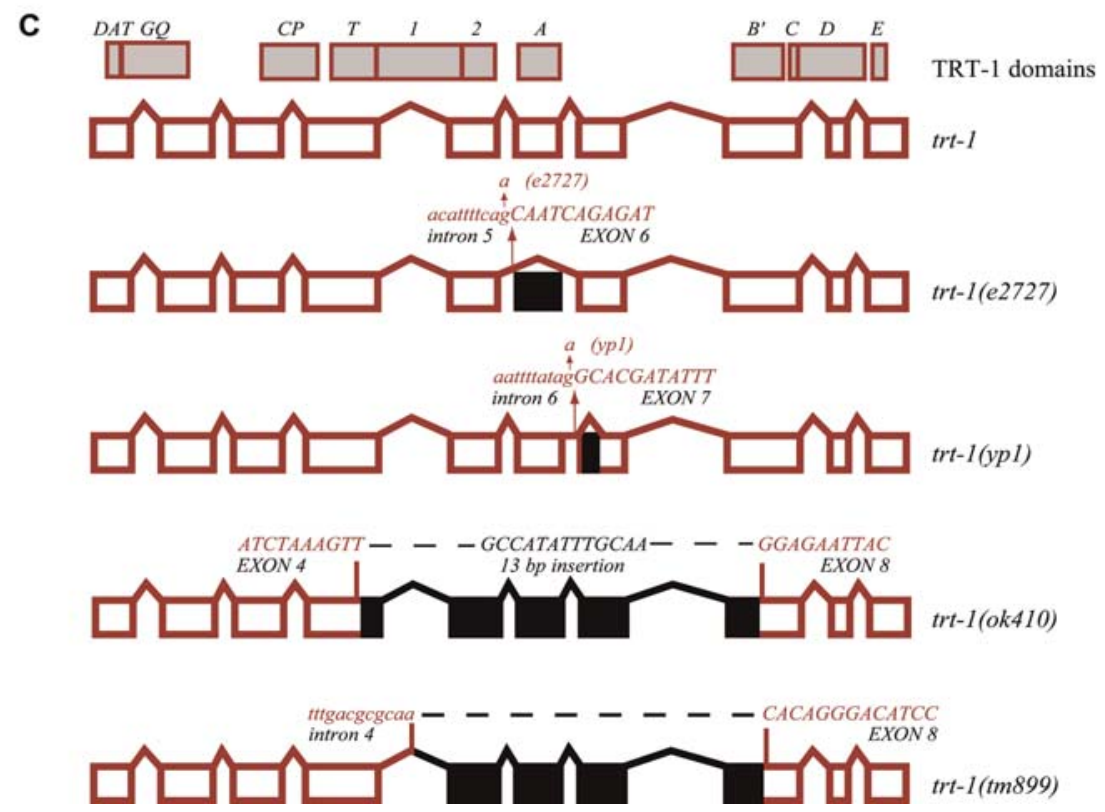

Figure 1. trt-1 Is the C. elegans Catalytic Subunit of Telomerase

(A) Three X-autosome fusions isolated from trt-1(e2727) display linkage between an end of the X chromosome and an end of an autosome. ypT3 and ypT16 were independent chromosome fusions isolated from parallel lines grown from the same F2 founder, suggesting that the two fusiogenic chromosome ends may correspond to short telomeres in the F2 founder, recapitulating our previous observations regarding strains inheriting a short left end of the $\mathrm{X}$ chromosome in mrt-2 mutants [12] and supporting a mouse study of telomere length and genome instability in the absence of the telomerase RNA [61].

(B) End-to-end fusions in oocytes of late-generation trt-1 mutant worms. Two DAPI-stained oocyte nuclei are shown per picture as indicated by dashed circles.

(C) trt-1 gene structure with mutations. Exon-intron structures and TERT protein domains are shown. Effects of mutations for all four trt-1 alleles are shown as determined by genomic DNA and CDNA sequencing. Black boxes are used to depict exons missing as a consequence of trt- 1 splice-junction mutations or sequences eliminated by trt-1 deletions. For yp 1, intron 6 is retained and a cryptic intron in exon 7 becomes active. Sequences flanking each breakpoint are shown for trt-1 deletions. A 13-bp in-frame insertion occurred at the breakpoint of trt-1(ok410). Sequencing of cDNA from trt1 (tm899) indicated that the deletion results in a premature stop codon.

DOI: 10.1371/journal.pgen.0020018.g001

fusions ( $n=38$ and 48, respectively), whereas $67 \pm 10 \%$ of nonrescued $e 2727$ and $o k 410$ siblings displayed chromosome fusions ( $n=30$ and 40, respectively) (Figure 2C). Thus, the telomere replication defects of $e 2727$ and $o k 410$ were caused by mutation of the DY3.4 gene, which was therefore designated trt-1 (telomerase reverse transcriptase-1). Given that microinjection of trt-1 mutants with linearized DNA fragments yielded extrachromosomal arrays that were used for rescue assays, formation of these transmissible genetic elements does not require telomerase activity, suggesting that they may be circular or that they may adopt a linear structure with noncanonical telomeres.

Taken together, the above results indicate that $e 2727, y p 1$, tm899, and $o k 410$ are alleles of $t r t-1$, the C. elegans catalytic subunit of telomerase. Although our genetic evidence strongly suggests that trt-1 is the C. elegans catalytic subunit of telomerase, confirmation that trt-1 is required for telomerase activity in vitro will be important. However, such biochemical assays have yet to be established for C. elegans, despite significant efforts in this regard (L. Harrington, personal communication).

\section{trt-1 Encodes a Compact TERT}

C. elegans trt- 1 is predicted to encode only 540 amino acids and is one of the shortest TERT proteins known [16] (Figure $3 \mathrm{~A})$. The trt-1 gene is tightly flanked by the neighboring genes DY3.3 and DY3.5 (http://www.wormbase.org), and RT-PCR analysis confirmed the predicted polyadenylation site at its $3^{\prime}$ end and that trt-1 is trans-spliced to an SL2 spliced leader at its initiation methionine codon (unpublished data), as expected from whole genome analysis of SL2-spliced genes [17]. The domain structure of and a set of core amino acids in the seven 
A

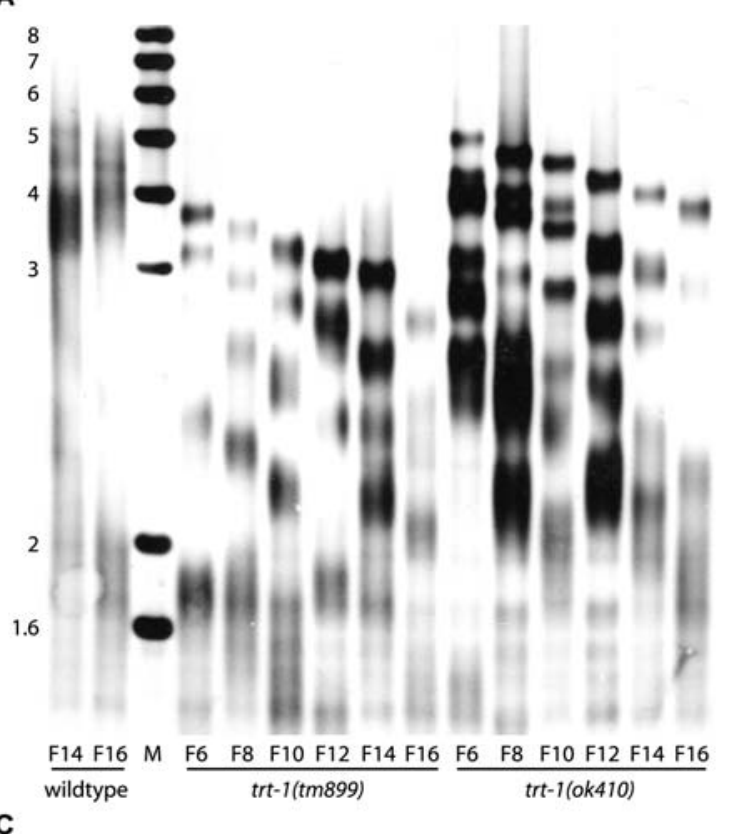

B

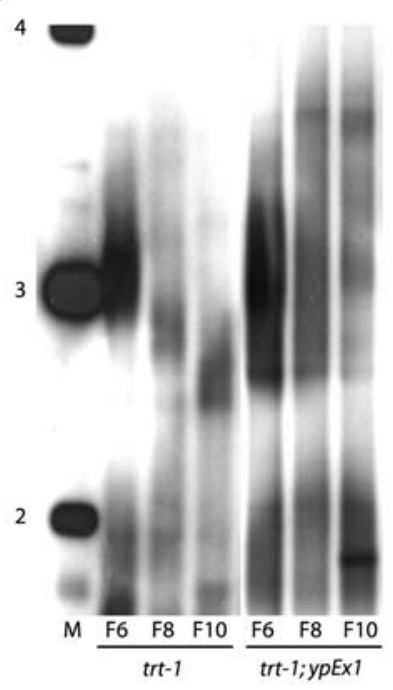

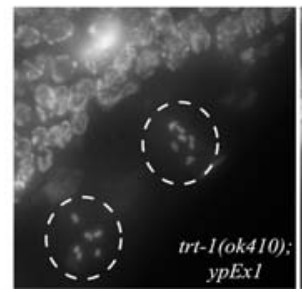
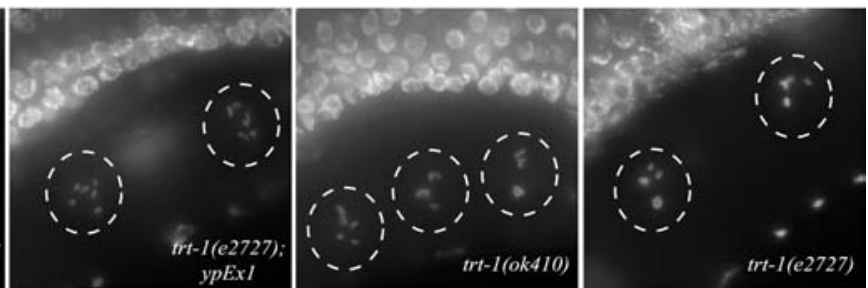

Figure 2. Progressive Telomere Shortening in trt-1 Mutants and Rescue by the Wild-Type DY3.4 Gene

(A) The trt-1 alleles tm899 and ok410 displayed comparable rates of telomere shortening over progressive generations. Two generations of N2 wild-type are shown for comparison. Southern blotting was performed using a (TTAGGC) probe and genomic DNA prepared from propagated strains as described [12].

(B and C) Expression of DY3.4 on the ypEx1 extrachromosomal array (B) eliminated telomere shortening for trt-1(ok410) in comparison with a nonrescued control and (C) repressed the formation of chromosome fusions observed in late-generation ok410 and e2727 mutants. Adult animals were stained with DAPI at generation F10, where nonrescued siblings exhibited a strongly reduced brood size or sterility. For Southern blots, sizes of DNA fragments are indicated to the left $(\mathrm{kb})$.

DOI: 10.1371/journal.pgen.0020018.g002

canonical reverse transcriptase motifs found in all reverse transcriptases were sufficient to identify TRT-1 as a TERT protein [16] (Figure 3B). In addition, TERT proteins have a number of unique motifs that distinguish them from other reverse transcriptases [18-20], including motif $\mathrm{T}$, which occurs immediately $\mathrm{N}$-terminal to the canonical reverse transcriptase motifs. Conserved N-terminal TERT motifs were not recognizable for C. elegans TRT-1 using BLAST analysis, indicating significant evolutionary divergence [16]. However, comparison of the predicted TRT-1 polypeptide of C. elegans with those of the related nematodes C. briggsae and C. remanei revealed considerable $\mathrm{N}$-terminal sequence homology (Figure S2A). When the N-termini of Caenorhabditis TRT-1 proteins were aligned with groups of TERT proteins from vertebrates, yeasts, or ciliates, homology with the DAT/I, GQ/I, and CP/II Nterminal motifs was apparent [18-20] (Figure 3, Figures S2B and $\mathrm{S} 2 \mathrm{C}$ ). In addition, an unusual motif $\mathrm{T}$ that lacked several highly conserved amino acids was observed for the Caenorhabditis genus, whose last common ancestor lived 100 million years ago. The more slowly evolving filarial parasitic nematode Brugia malayi, which diverged from C. elegans roughly 300 million years ago, had a motif $\mathrm{T}$ with greater similarity to those found in canonical TERT proteins (Figure 3B).

The last canonical reverse transcriptase motif of TERT, motif E, is typically followed by a telomerase-specific Cterminal domain of 150 to 200 amino acids, which harbors motifs that are essential for catalytic activity of telomerase in vitro and in vivo, for multimerization or processivity [21-23]. However, Caenorhabditis TRT-1 proteins had a C-terminal domain of less than 30 amino acids, which lacked substantial homology to any of the phylum-specific conserved C-terminal motifs found in other TERT proteins [16] (Figure 3 and unpublished data). Thus, Caenorhabditis TRT-1 has diverged substantially at both its $\mathrm{N}$ - and C-termini.

\section{trt-1 and the mrt-2 DNA Damage Checkpoint Gene Function in the Same Pathway}

Mutations in the $\mathrm{Ku}$ heterodimer result in short but stable telomeres in Saccharomyces cerevisiae and Schizosaccharomyces pombe [24-27]. Yeast strains that contain mutations in both $\mathrm{Ku}$ and telomerase display faster telomere shortening and senescence phenotypes than do strains harboring a single telomerase mutation [25,27]. A synthetic phenotype between 


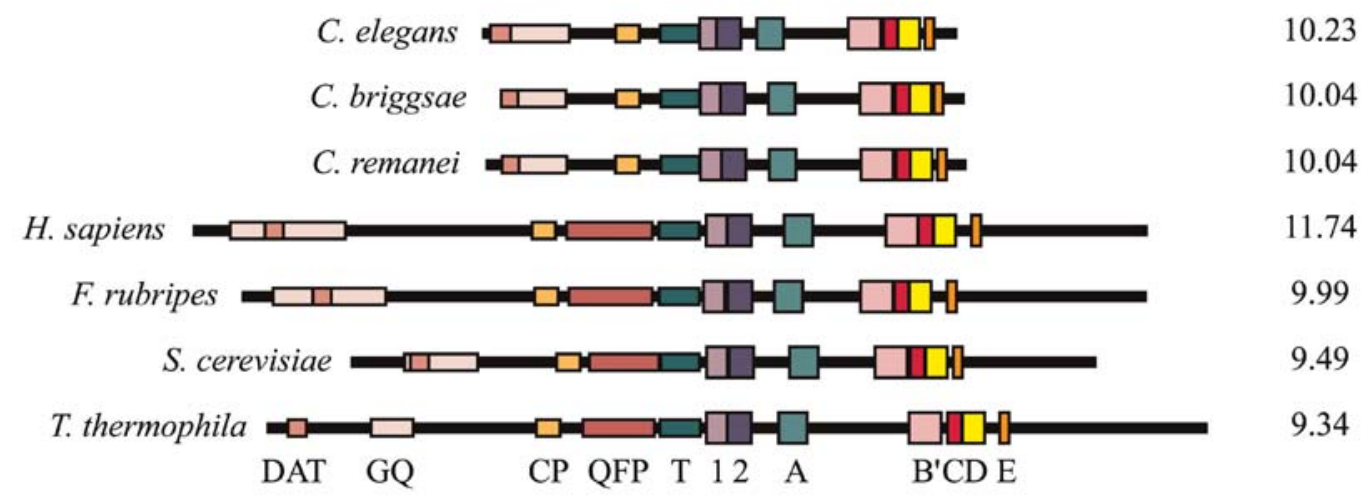

B

Motif DAT

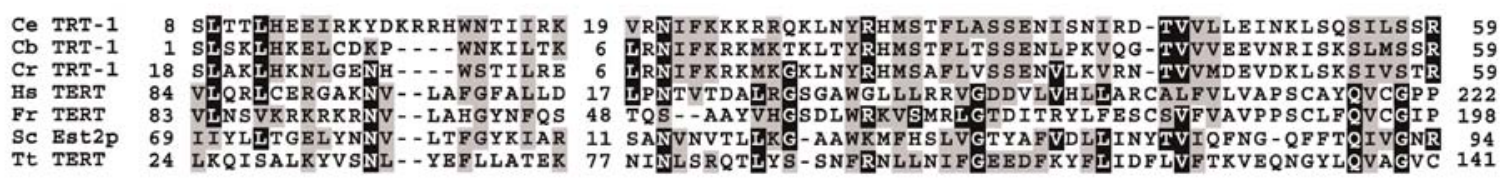

Motif II/CP

Ce TRT-1

Cb TRT-

Cr TRT-

Hs TERT

Fr TERT

Sc Bst2p

Tt TERT

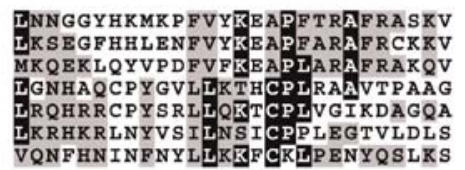

Motif 1

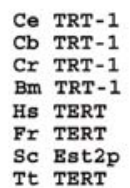

$\begin{array}{ll}\text { Ce } & \text { TRT-1 } \\ \text { Cb } & \text { TRT-1 } \\ \text { Cr } & \text { TRT-1 } \\ \text { Bm } & \text { TRT-1 } \\ \text { Hs } & \text { TERT } \\ \text { Fr } & \text { TERT } \\ \text { Sc } & \text { Est2p } \\ \text { Tt } & \text { TERT }\end{array}$
Motif $\mathrm{T}$

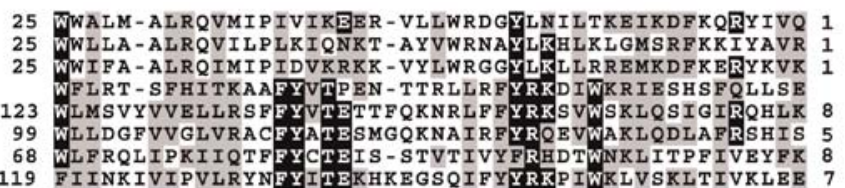

Motif 2

Motif A

Ce TRT-1

Cb TRT-1

$\begin{array}{ll}\text { Cr } & \text { TRT- } \\ \text { Hs } & \text { TBRT }\end{array}$

$\begin{array}{ll}\text { Hs } & \text { TBRT } \\ \text { Fr TERT }\end{array}$

$\begin{array}{ll}\text { Fr } & \text { TBRT } \\ \text { Sc } & \text { Est }\end{array}$

Tt TERT

APHFIR- - - PNV - - ATFKLSISRQK 0

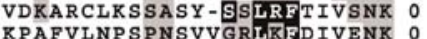
KPAFVLNPSPNSVVGRLKFDIVENK 0
EVRQHREARPALLTSRLRFIPKPDG 0 $\begin{array}{ll}\text { TPDQVAALPKSTIISRLRFIPKTDG } & 4 \\ \text { CRNHNSYTLSNFNHSKMRIIPKIKN } & 2\end{array}$ CRNHNSYTLSNFNHSKMRIIPKKSN 2
BKLIPEDSFQKYPQGKLRIIPKIKGS 0

Motif $B^{\prime}$

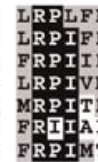

Motie 2

$\begin{array}{llll} & \end{array}$ $\begin{array}{llll} & \end{array}$ $\begin{array}{lllll} & \end{array}$ $\begin{array}{llll} & \\ \text { PITRVIGADAKTRLYQSHVRDLLDMLR } & 33 & \text { KPQPLYTVRMDVSGAYESLPENKLIEVINQVITP } & 91\end{array}$ IIAIPCRGADEREFTIYKENHKNAIQP 72 VLPELYFMKFDVKSCYDSIPRMECMRILKDALKN 68

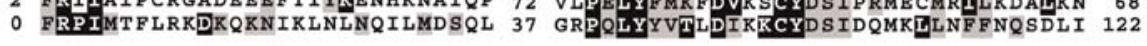

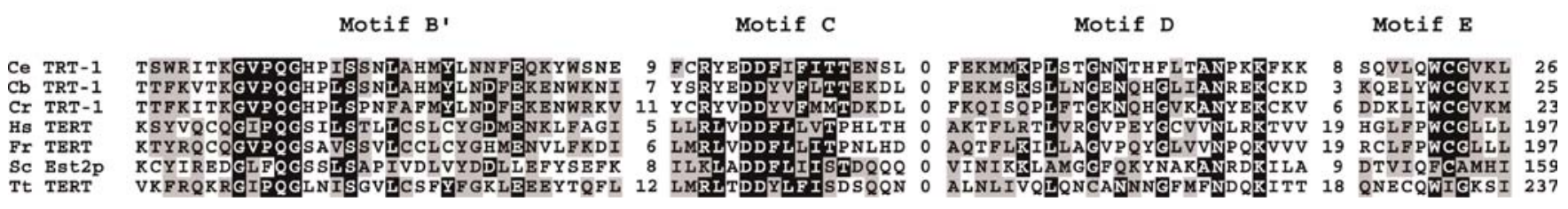

Figure 3. Structure and Sequence Motifs of Caenorhabditis TERT Proteins

(A) Structure of TERT proteins from distantly related species, with isoelectric point (pl) indicated.

(B) TERT sequence motifs include seven canonical reverse transcriptase motifs (1, 2, A, B', C, D, and E) and several TERT-specific N-terminal motifs (I/DAT/ GQ, II/CP, and T) [18-20] for TERT proteins from three Caenorhabditis species; C. elegans (Ce), $\mathrm{C}$. briggsae (Cb), and C. remanei (Cr); from the vertebrates $H$. sapiens (Hs) and Fugu rubripes (Fr); and from Saccharomyces cerevisiae (Sc) and Tetrahymena thermophila (Tt). An additional motif T is included for the nematode Brugia malayi $(\mathrm{Bm})$, as this sequence was critical for precisely defining the Caenorhabditis motif T. Only portions of motif I/GQ and motif DAT with homology to the Caenorhabditis TERT proteins are shown. Motif DAT is a specialized protein domain within motif I/GQ that is known to be required for telomerase activity in vivo [42]. A clearly defined III/QFP N-terminal motif was not identified for the Caenorhabditis TERTs. The Brugia malayi T motif is based on a short expressed sequence tag, and further N-terminal motifs could not be discerned from the current genome sequence (http://www.tigr. org). Sequence alignments were performed using Pole Biolnformatique Lyonnais ClustalW 1.8 (http://pbil.ibcp.fr/htm/index.php).

DOI: 10.1371/journal.pgen.0020018.g003

$\mathrm{Ku}$ and telomerase has also been reported for Arabidopsis [28]. Therefore, $\mathrm{Ku}$ is thought to affect telomere integrity via a telomerase-independent mechanism, perhaps by protecting telomeres from exonucleolytic attack [29]. In contrast, $S$. cerevisiae Mre11/Rad50/Xrs2 nuclease complex mutants have short but stable telomeres, but in this case double mutants defective for Mre11/Rad50/Xrs2 and the telomerase reverse transcriptase display the same telomere erosion and senescence phenotypes as telomerase mutants, arguing that these genes may function in the same telomere replication pathway $[25,30]$.

Null mutations in two subunits of the heterotrimeric 9-1-1 DNA damage response complex, mrt-2 and hus-1, result in telomere erosion and late-onset end-to-end chromosome 
A

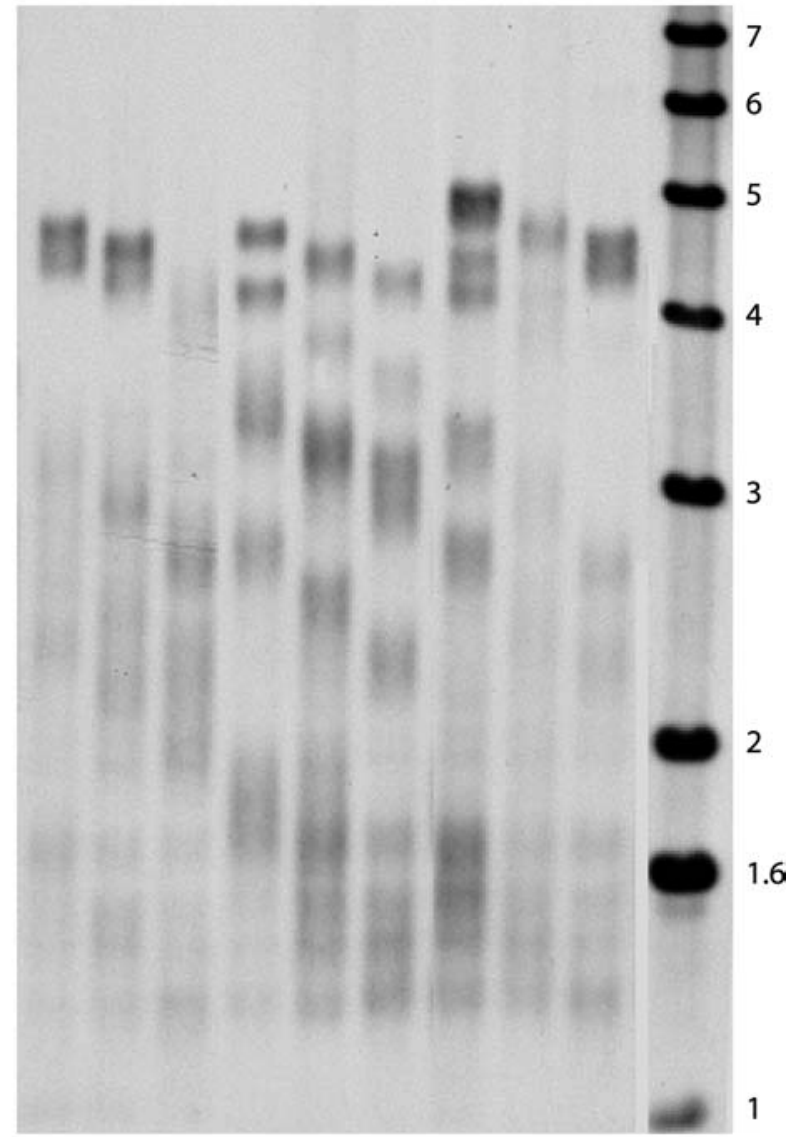

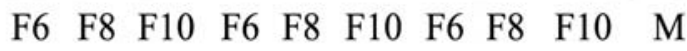

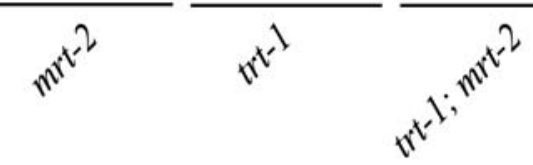

B

\section{$\begin{array}{lllllllll}\text { F4 } & \text { F6 } & \text { F8 } & \text { F10 } & \text { F12 } & \text { F14 } & \text { F16 } & \text { F18 }\end{array}$}

$\begin{array}{rcccccccc}m r t-2 . a & \mathrm{~W} & \mathrm{~W} & \mathrm{~W} & \mathrm{M} & \mathrm{F} & \mathrm{S} & & \\ m r t-2 . b & \mathrm{~W} & \mathrm{~W} & \mathrm{~W} & \mathrm{M} & \mathrm{M} & \mathrm{F} & \mathrm{S} & \\ t r t-1 . a & \mathrm{~W} & \mathrm{~W} & \mathrm{~W} & \mathrm{M} & \mathrm{F} & \mathrm{F} & \mathrm{S} & \\ t r t-1 . b & \mathrm{~W} & \mathrm{~W} & \mathrm{~W} & \mathrm{~W} & \mathrm{M} & \mathrm{M} & \mathrm{F} & \mathrm{S} \\ t r t-1 ; m r t-2 . a & \mathrm{~W} & \mathrm{~W} & \mathrm{~W} & \mathrm{~W} & \mathrm{M} & \mathrm{F} & \mathrm{S} & \\ t r t-1 ; m r t-2 . b & \mathrm{~W} & \mathrm{~W} & \mathrm{~W} & \mathrm{~W} & \mathrm{M} & \mathrm{F} & \mathrm{F} & \mathrm{S}\end{array}$

Figure 4. trt-1 and mrt-2 Function in the Same Pathway

(A) Progressive telomere shortening of $m r t-2$ single, trt-1(tm899) single, and trt-1(tm899); $m r t-2$ double mutant strains.

(B) Comparison of late onset of sterility in mrt-2 single, trt-1(tm899) single and $t r t-1$ (tm899); $m r t-2$ double mutant strains. Two independent strains, a and $b$, are shown for each genotype. Plates were seeded with six L1 animals, and the brood size was scored after 7 days of growth as wildtype (W), medium (M), few (F), and sterile (S) [12]. Note that initial telomere lengths observed for these strains reflect random telomere segregation during a cross. Therefore, the rate of telomere erosion is a more significant measure of additivity than are differences in initial telomere length or the time to sterility. Sizes of DNA fragments are indicated to the right $(\mathrm{kb})$.

DOI: 10.1371/journal.pgen.0020018.g004 fusions in C. elegans [12,14], as observed for trt-1 mutants (Figures 1 and 2). To determine if the 9-1-1 complex and trt-1 act in the same pathway to facilitate telomere replication, rates of telomere erosion were measured for strains of trt1(tm899);mrt-2 double mutants and for trt-1(tm899) and mrt-2 single mutants, all of which were derived from F2 siblings of the same F1 parent, thereby ensuring that their initial telomere lengths would be as similar as possible. Rates of telomere shortening in these strains were $135 \pm 28 \mathrm{bp} /$ generation for trt-1(tm899), $133.4 \pm 34.7 \mathrm{bp} /$ generation for mrt-2, and $119.4 \pm 31.5 \mathrm{bp} /$ generation for trt-1(tm899); $m r t-2$ (Figure 4A; $n=2$ independent strains each), which did not significantly differ from each other or from the rates observed for other trt- 1 alleles (Figures 2A and S1). In addition, the number of generations to sterility for both trt1(tm899) and mrt-2 single mutants and for trt-1(tm899);mrt-2 double mutants was similar (Figure 4B). Identical results were observed with double mutant strains harboring mutations in $m r t-2$ and either $t r t-1(o k 410)$ or trt-1(e2727) ( $n=2$ for each $t r t-1$ allele, unpublished data). Thus, additivity was not observed for the rate of telomere erosion in trt-1;mrt-2 double mutants, indicating that telomerase and the 9-1-1 checkpoint complex that responds to DNA double-strand breaks may act in the same telomere replication pathway. This conclusion is based on the premise that a rate of telomere shortening of greater than $\sim 125$ bp per generation is possible.

Although the 9-1-1 complex may be required for trt-1 activity at chromosome termini, trt-1 displays p53-mediated, ionizing radiation-induced apoptosis (B. Meier and S. Ahmed, unpublished data), whereas this response is abrogated in the mrt-2 DNA damage response mutant [31]. Therefore, $t r t-1$ is not required for the 9-1-1 complex to respond to damage caused by ionizing radiation, such as DNA double-strand breaks.

\section{trt-1 Mutants Become Sterile as a Consequence of Mitotic Failure}

S. cerevisiae ever shorter telomeres mutants that are defective for telomere replication display a senescence phenotype in which the vast majority of cells die but "survivors" typically emerge that maintain their telomeres using homologous recombination [32]. Mammalian cells that lack telomerase can also survive telomere erosion by using a recombinationdependent process of telomere maintenance [33]. However, we have never observed survivors to arise for C. elegans trt-1 mutants, despite having grown hundreds of $t r t-1$ strains to sterility (unpublished data). Thus, telomerase-independent recombination-based telomere maintenance may be incompatible with meiosis in C. elegans, a notion supported by the lack of any reported survivors in mouse strains deficient for telomerase [34].

Levels of apoptosis increase dramatically in the germlines of late-generation mouse telomerase RNA mutants [34], presumably as a consequence of telomere uncapping, endto-end chromosome fusions, and/or subsequent breakagefusion-bridge cycles. Telomere uncapping has also been shown to elicit either apoptosis or senescence in mammalian cells [35]. The C. elegans germline normally incurs a low background level of physiological apoptosis, and increased rates of apoptosis occur in response to either ionizing radiation or inactivation of $\mathrm{rad}-51$ [31,36], treatments that result in DNA double-strand breaks. However, levels of apoptosis were unaltered for all four C. elegans trt-1 alleles 
Table 1. Basal Germline Apoptosis Is Normal in trt-1 Mutants at Early (F7) and Late (F15) Generations

\begin{tabular}{lll} 
Strain & \multicolumn{2}{l}{ Apoptotic Corpses } \\
\cline { 2 - 3 } & Early (F7) & Late (F15) \\
\hline Wild-type & & \\
trt-1(e2727) & $4.5 \pm 0.77$ & $3.5 \pm 0.43$ \\
trt-1(ok410) & $3.9 \pm 0.95$ & $4.5 \pm 0.13$ \\
trt-1(tm899) & $4.5 \pm 0.13$ & $4.9 \pm 0.22$ \\
trt-1(yp1) & $4.7 \pm 0.9$ & $4.2 \pm 0.8$ \\
& $3.5 \pm 0.34$ & $4.4 \pm 0.29$
\end{tabular}

L4 hermaphrodites were picked from wild-type and from early and late generations of e2727, yp 1, tm899, and ok410 mutants, and 1-d-old adults were stained for apoptosis using acridine orange [38]. Each strain was tested three times and, minimally, 16 gonad arms were scored per experiment. trt-1 mutants display an increasing number of animals with short germlines in late generations. Animals with visibly short an increasing number of animals with short germlines in late generations. Animals with visibly short
gonad arms were excluded from the analysis, as some had low levels of apoptosis that were probably a consequence of underproliferation. In very late generations where many animals were sterile, one in 30 to 40 germlines had an increased rate of apoptosis, but this did not significantly affect the overall levels of apoptosis. Data are shown as mean \pm SD.

DOI: 10.1371/journal.pgen.0020018.t001

in later generations, as compared to early generations or to wild-type controls (Table 1).

Given that late-generation $t r t-1$ mutants did not experience increased levels of apoptosis, sterile trt-1 mutants were examined to assess the cause of their germline proliferation defects. C. elegans rad-51 mutants display relatively normal levels of germ cell proliferation but lay hundreds of embryos that die as a consequence of aneuploidy and genome instability [37,38]. In contrast, few if any embryos were laid by trt- 1 hermaphrodites as they approached sterility, indicating that these mutants experience progressive impairment of germline proliferation (unpublished data). Several developmental defects were detectable in late-generation trt-1 adults, such as a Protruding Vulva, a weak Uncoordinated phenotype, small worms with Body Morphology Defects, small sickly males, and occasional Slow Growth (unpublished data). A large percentage of trt-1 mutant animals had short germline arms that ranged from medium size to an empty gonad (Figure 5A-5D). Gonad arm size often differed within a single animal, suggesting a stochastic germline proliferation defect, as might be expected for telomere replication mutants. High-resolution microscopy of sterile $t r t-1$ germlines revealed a variety of other defects, including masculinization of the germline, abnormal oocyte formation, endomitotic oocytes, and lack of sperm (Figure 5E-5I). In addition, chromatin bridges were observed between intestinal and embryonic nuclei of trt-1 mutants but not between nuclei of wild-type controls (Figure 5J and 5K and unpublished data). Similar phenotypes have been reported previously for knockdown of $C$. elegans kinetochore components by mutation or RNA interference (http://www.wormbase.org) [39]. To confirm this possibility, we examined hermaphrodites defective for the him-10 kinetochore gene [40], which incur high rates of mitotic chromosome nondisjunction. A spectrum of phenotypes similar to that found in sterile trt-1 mutants was observed (Figure 5L), suggesting that trt- 1 mutants may become sterile as a consequence of chromosome segregation defects.

\section{Neither trt-1 nor Telomere Length Affects C. elegans Life Span}

Many normal human somatic cells repress TERT expression and lack significant levels of telomerase activity [1,2], properties that result in telomere erosion and may contribute to aspects of the aging process. As mutation of trt- 1 ought to mimic the telomerase deficiency observed in human somatic cells, we sought to determine if either trt-1 or telomere erosion might affect the aging process in C. elegans. Initial experiments were conducted with trt-1(e2727) or trt-1(ok410) strains, where early- and middle-generation strains were starved for several weeks prior to conducting life span analysis of early-, middle-, and late-generation strains in parallel. These studies suggested that neither trt-1 nor telomere erosion affects life span in C. elegans (unpublished data). In order to control for any effects that extended starvation might have had on the early- and middlegeneration samples described above, outcrossed strains homozygous for the trt-1 deletion ok410 were passaged, frozen at early-, middle-, and late-generations, and then thawed for life span analysis together. No change in life span was observed between wild-type (19.9 \pm 0.6 days) and early- (20.6 \pm 0.6 days $)$, mid- (20.4 \pm 0.6 days), or late- $(20.0 \pm 0.6$ days $)$ generations of a $\operatorname{trt}-1(\mathrm{ok} 410)$ strain $(P=0.18,0.22$, and 0.46 , respectively), where telomeres of mid- and late-generation strains were about 1 and about 1.5 kilobases shorter than those of early-generation strains, respectively (Figure 6). Experiments with an additional allele of trt- 1 confirmed that life spans of N2 wild-type (19.1 \pm 0.4 days) and early- (19.3 \pm 0.5 days $)$ or late- (18.3 \pm 0.5 days $)$ generations of a trt-1(tm899) strain were not significantly different $(P=0.96$ and 0.23 , respectively) (Figure 6). Similar results were obtained with independently derived $o k 410$ and $\operatorname{tm} 899$ strains (Figure S3). The late-generation $t r t-1$ strains used for the above life span experiments displayed significant drops in brood size as a consequence of critically shortened telomeres being fused end-to-end (unpublished data). Thus, our results suggest that neither trt-1 nor telomere erosion in the absence of trt- 1 nor chromosome uncapping and end-to-end fusion affects $C$. elegans life span.

\section{Discussion}

Here we genetically define the C. elegans catalytic subunit of telomerase, trt-1, and study the effects of telomere erosion in trt-1 mutants. TRT-1 is one of the smallest TERT proteins known as a consequence of truncated $\mathrm{N}$ - and C-terminal TERT-specific domains [16] (Figure 3). Functions known to be conferred by N- and C-termini of TERT include recruitment of telomerase to chromosome ends, nucleolar localization, and TERT multimerization [21-23]. Further, N-terminal domains of Tetrahymena, S. cerevisiae, and human TERT, including motif $\mathrm{T}$, have been shown to mediate physical interactions with the telomerase RNA [18,41-43], so the divergent Caenorhabditis TERT N-terminus might reflect a telomerase RNA with unusual structural qualities. Consistent with this possibility, the C. elegans telomerase RNA has yet to be discovered in searches for noncoding RNAs with a telomeric template and secondary structures similar to those found in vertebrate telomerase RNAs [44], despite the availability of two fully sequenced Caenorhabditis genomes and extensive $C$. elegans cDNA sequencing $[45,46]$. Thus, TRT- 1 has an unusual primary structure that may suggest functional divergence.

Mutation of yeast DNA damage checkpoint proteins typically results in shortened telomeres, whereas double mutants that lack both of the functionally redundant damage 

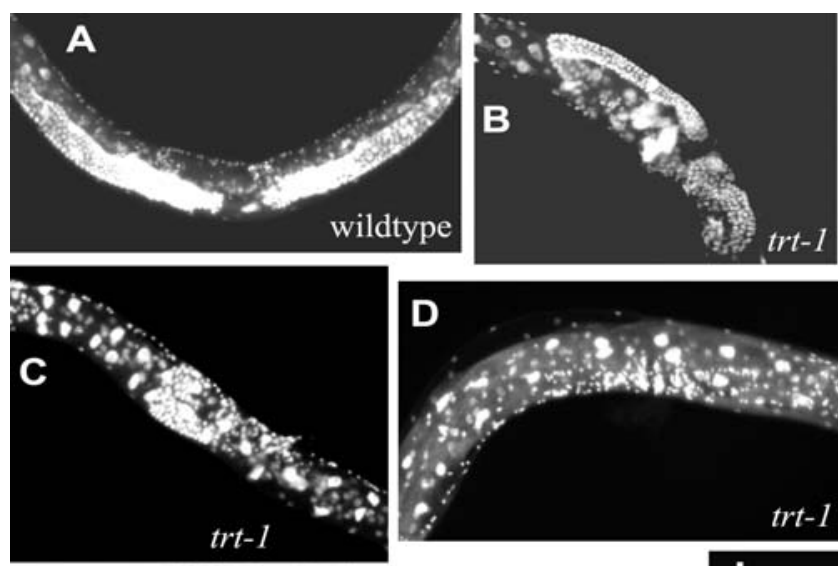

wildtype
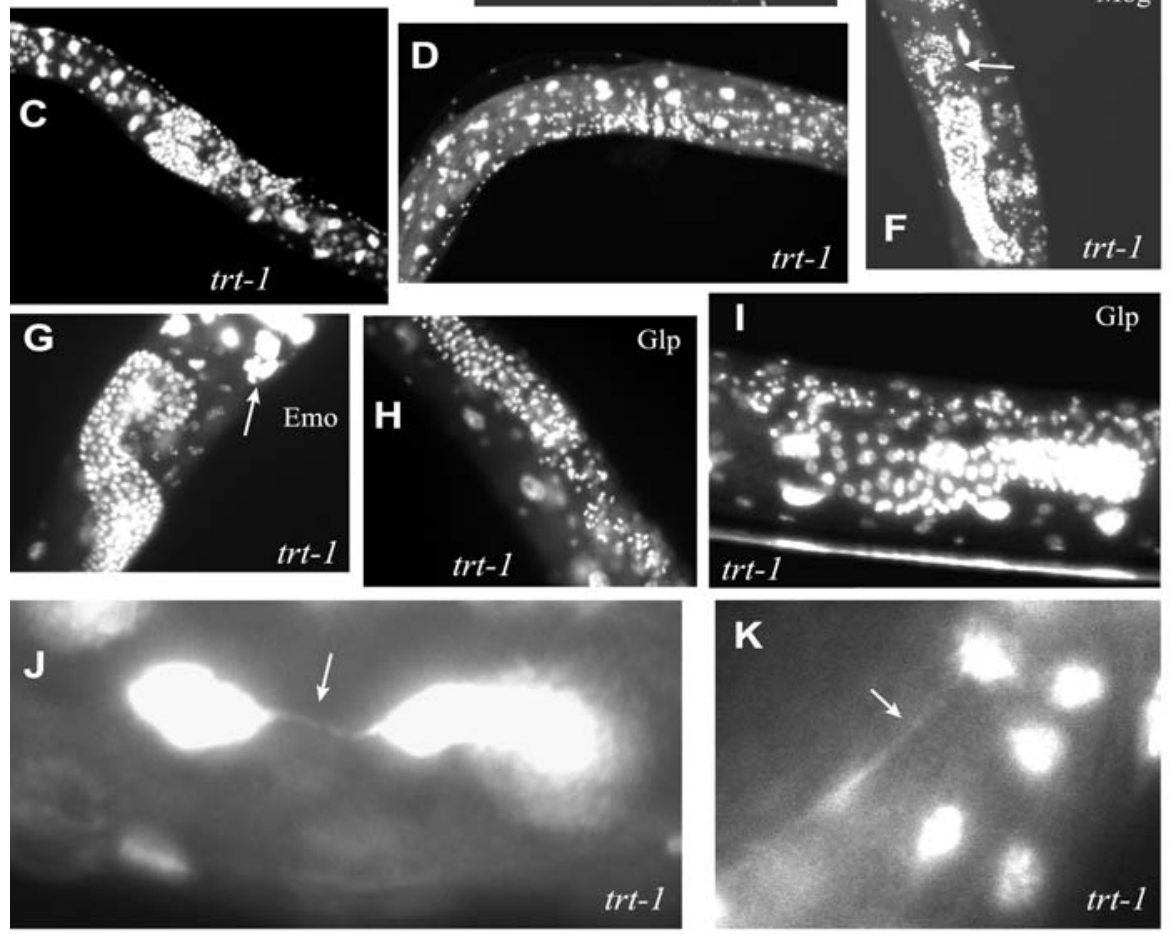

\begin{tabular}{l|ll|ccc|cccc}
\multicolumn{2}{c}{} & \multicolumn{4}{c|}{ Somatic } & \multicolumn{5}{c}{ Germline } \\
\multicolumn{3}{c}{ Strain } & Pvl & Vul & Egl & Ooc & Emo & Mog & Glp \\
\hline \multirow{3}{*}{$20^{\circ} \mathrm{C}$} & wildtype & $(\mathrm{n}=25)$ & $0 \%$ & $0 \%$ & $0 \%$ & $0 \%$ & $0 \%$ & $0 \%$ & $0 \%$ \\
& trt-1(ok410) & $(\mathrm{n}=50)$ & $8 \%$ & $10 \%$ & $10 \%$ & $9 \%$ & $9 \%$ & $21 \%$ & $17 \%$ \\
& trt-1(tm899) & $(\mathrm{n}=50)$ & $14 \%$ & $4 \%$ & $6 \%$ & $9.3 \%$ & $15.5 \%$ & $16.5 \%$ & $22.7 \%$ \\
\hline $25^{\circ} \mathrm{C}$ & him-10 & $(\mathrm{n}=24)$ & $0 \%$ & $4.2 \%$ & $12.5 \%$ & $10.4 \%$ & $6.3 \%$ & $6.3 \%$ & $16.7 \%$
\end{tabular}

Figure 5. trt-1 Mutants Display Mitotic Defects at Sterility

(A) Wild-type germlines displayed two symmetric, highly proliferative arms of the germline when stained with DAPI.

(B-K) In contrast, trt-1 mutants at sterility displayed medium and small germline arms (B), small and empty germline arms (C), and only empty germline arms (D). Some trt-1 mutants had a Masculinization of the Germline (Mog) phenotype and only produced sperm (E and F), many displayed endomitotic oocytes that endoreduplicate in the absence of sperm (G), some germlines displayed an abnormal Germline Proliferation (Glp) phenotype where the few germ cells that were produced were mitotic ( $\mathrm{H}$ and $\mathrm{I})$, and anaphase bridges were observed between intestinal nuclei in sterile trt-1 adults ( $\mathrm{J}$ and $\mathrm{K}$ ) but not in wild-type controls.

(L) Quantification of somatic and germline phenotypes in wild-type, in trt-1 mutants, and in the kinetochore protein mutant him-10 grown at the restrictive temperature of $25^{\circ} \mathrm{C}$ [40]. Phenotypes scored were Protruding Vulva (Pvl), Vulvaless (Vul), Egg-Laying Defective (Egl), Intestinal Nuclear Bridge (lbr), Abnormal Oocyte Formation (Ooc), Endomitotic Oocyte (Emo), Mog, and Glp. Although the Pvl phenotype was not observed in him-10 animals, it is commonly observed for depletion of other kinetochore components by RNA interference (http://www.wormbase.org).

DOI: 10.1371/journal.pgen.0020018.g005

signaling proteins Mec1p/ATR and Tel1p/ATM have an Ever Shorter Telomeres phenotype that is similar to that observed for yeast telomerase mutants $[47,48]$. Thus, redundant DNA damage signaling is required for telomere replication in yeast. Since disruption of telomere structure by several means allows telomerase to act at telomeres in the absence of both Mec1p and Tellp, the telomere-specific role of damage checkpoint signaling in yeast may be one of mediating telomerase access [49]. Two C. elegans orthologs of subunits of the 9-1-1 DNA damage response complex, mrt-2 and hus-1, are required for telomere replication in C. elegans [12,14], whereas mutation in their S. cerevisiae or $S$. pombe orthologs results in short but stable telomeres [50,51]. We show here that $m r t-2$ and $t r t-1$ may act in the same pathway (Figure 4), a possibility that may be difficult to assess in mammals, where the 9-1-1 complex is essential [52]. Given that the 9-1-1 complex can be loaded onto aberrant DNA structures at double-strand breaks and stalled replication forks [53], it may 
A

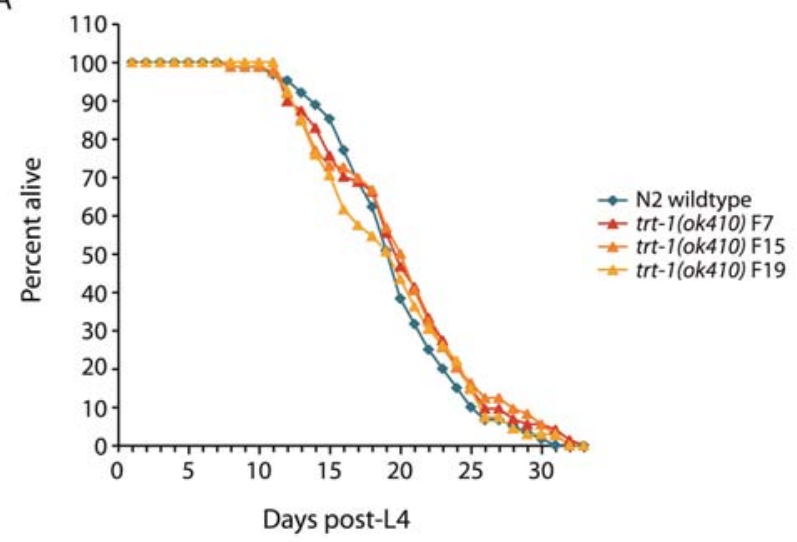

B

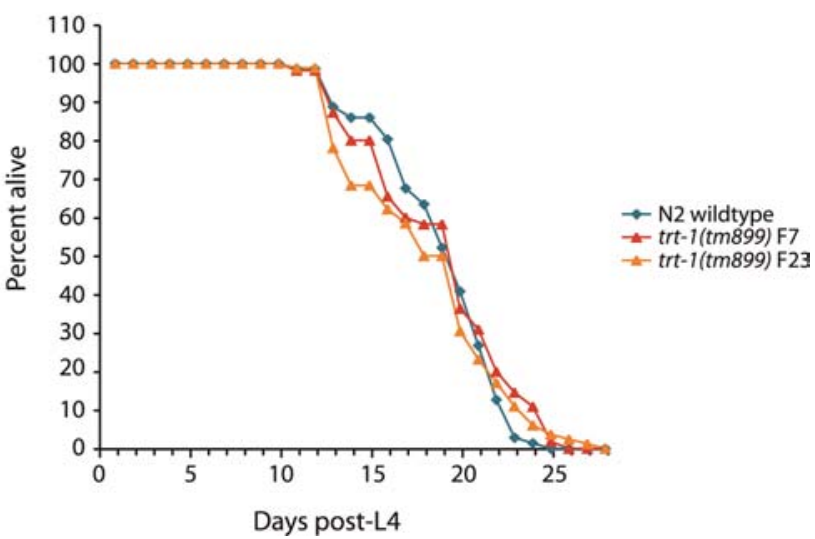

Figure 6. trt-1 Mutants Have a Normal Life Span Independent of Telomere Length and Telomere Uncapping

(A) Life spans of N2 wild-type and early, middle, and late generations of a trt-1(ok410) strain. Mutants were analyzed at generations with slightly shortened telomeres (F7), short telomeres (F15), and very short telomeres with chromosome fusions (F19).

(B) Life spans of N2 wild-type and early and late generations of a trt1 (tm899) strain.

DOI: 10.1371/journal.pgen.0020018.g006

respond to replicating telomeres during $\mathrm{S}$ phase and assist with checkpoint-mediated modification or turnover of core telomere protein components. Thus, the redundant Mec1p/ ATR and Tellp/ATM checkpoint signaling pathways that function in yeast telomere replication may have been replaced by a single pathway in C. elegans. Alternatively, redundant checkpoint signaling may be required for mediating telomerase access in C. elegans, but the 9-1-1 complex may have an additional, essential role in facilitating telomerase activity.

In contrast to late-generation mouse telomerase RNA mutants [34], late-generation trt-1 mutants failed to display an overall increase in apoptosis (Table 1), despite being proficient for p53-mediated, DNA damage-induced apoptosis (B. Meier and S. Ahmed, unpublished data). One explanation for this discrepancy might be that end-to-end chromosome fusions that result from telomere attrition in monocentric organisms such as mice and humans break frequently, which may elicit an apoptotic response. In contrast, C. elegans has holocentric chromosomes that can be propagated stably when fused, perhaps affording protection from break-induced apoptosis [54]. Although massive telomere uncapping can elicit an apoptotic response in mammalian cells [35], it is unclear how many DNA double-strand breaks are required to induce apoptosis in the C. elegans germline. Given that individual telomeres of a trt-1 strain are likely to have different initial telomere lengths, only a small proportion of the 24 telomeres of a C. elegans trt- 1 mutant are likely to be uncapped at any point in time, even in later generations. Our data indicate that if uncapped telomeres are sensed as doublestrand breaks in C. elegans, then the resulting DNA damage signaling is not sufficient to trigger apoptosis in trt-1 mutants.

Levels of apoptosis were not enhanced in C. elegans trt-1 mutants near sterility, but several phenotypes indicative of mitotic failure were observed (Figure 5). Given that trt-1 mutants have typically incurred two or three end-to-end chromosome fusions by this time, subsequent fusion events may involve chromosome circularization. C. elegans has holocentric chromosomes that may interfere with mitotic segregation of circular chromosomes. Accordingly, the anaphase bridges observed between intestinal nuclei in lategeneration trt-1 mutants may reflect the presence of circularized chromosomes, which have never been recovered in C. elegans. Although circular chromosomes are mitotically stable in the monocentric yeast $S$. pombe, meiosis fails in the presence of such chromosomes [47]. Thus, chromosome circularization may result in mitotic as well as meiotic failure in late-generation trt-1 mutants.

Linear end-to-end chromosome fusions consisting of two chromosomes typically display stable mitotic and meiotic transmission in C. elegans [12] (Figure 1A). In contrast, the only triple end-to-end fusion that has been constructed appears to break or mis-segregate frequently [55], and instability associated with multiply fused chromosomes may account for some of the phenotypes observed in late-generation trt-1 mutants. For example, mitotic loss of a significant portion of the $\mathrm{X}$ chromosome during development of the germline could result in a Masculinization Of the Germline phenotype, which was displayed by sterile trt-1 mutants and by mutants defective for chromosome segregation [39] (Figure 5). Other developmental phenotypes that occurred in sterile trt-1 mutants, such as a Protruding Vulva, Uncoordinated neuromuscular defects, and occasional Slow Growth, have also been observed in C. elegans strains with chromosome segregation defects [39] (http://www.wormbase.org) (Figure 5). In summary, sterile trt-1 mutants displayed a variety of phenotypes indicative of chromosome mis-segregation and/or mitotic failure, which may be the ultimate cause of sterility.

Some aspects of mammalian proliferative aging appear to be regulated by telomere erosion [6-10], but it is unclear if telomere length also affects postmitotic aging or whether the downregulation of TERT itself in human somatic cells might affect either proliferative or postmitotic aging by a mechanism that is independent of telomere length [56]. No difference in life span was observed between wild-type $C$. elegans strains and trt-1 mutants with either long or short telomeres, even when critically shortened telomeres were present (Figures 6 and S3). These results suggest that neither telomere length nor TERT affects postmitotic aging of the soma in C. elegans, although proliferative immortality of the germline is clearly compromised. Thus, C. elegans TERT plays a role in proliferative rather than postmitotic aging. The presence of end-to-end fusions did not affect trt- 1 life span, but significant amounts of subtelomeric DNA are lost at the fusion breakpoints of some C. elegans end-to-end fusions (M. 
Lowden and S. Ahmed, unpublished data). Such sequence loss might occasionally compromise life span for some lategeneration $t r t-1$ strains, although we failed to observe effects on life span in trt-1 strains with multiple end-to-end fusions (Figures 6 and S3). A recent report suggests that modest increases in telomere length might extend postmitotic life span in C. elegans [57]. In this instance, it is possible that elongated telomeres correlated with extended life span but were not causal and that the observed effects on life span could have been due to another experimental variable. Indeed, a recent study of various wild strains of $C$. elegans failed to observe a correlation between significantly elongated telomeres and life span [58]. While this complements our observations that telomere shortening has no effect on life span for trt-1 mutants, the relationship between elongated telomeres and life span in C. elegans will remain equivocal until strains with normal and significantly elongated telomeres are studied in isogenic backgrounds. We conclude that although telomere erosion in C. elegans trt-1 mutants effectively mimics telomere dysfunction experienced by most human somatic cells, life span is not altered in these mutants. trt-1 mutants may instead be useful for studying other properties of telomerase, such as its role in the development of cancer [59].

\section{Materials and Methods}

Strains and plasmids. Strains were cultured at $20{ }^{\circ} \mathrm{C}$ as described [60], unless stated otherwise. The following mutant strains were used: trt-1(e2727), trt-1(yp1), trt-1(tm899), trt-1(ok410), mrt-2(e2663), him10(e1511), unc-13(e51)dpy-24(s71), and unc-29(e193). trt-1;mrt-2 double mutants were generated by crossing unc-29;mrt-2 hermaphrodites with trt-1+l+unc-29 males. mrt-2 F2 homozygotes were identified based on radiation hypersensitivity, whereas trt-1 homozygotes were identified based on linkage with unc-29. The plasmid pSA1, which comprises the trt-1 open reading frame as well as upstream and downstream sequences, was generated by digestion of cosmid H14N5 (Alan Coulson, personal communication) with EaeI and ligation into the NotI site of pBS-KSII ${ }^{+}$.

RNA preparation and RT-PCR. RNA was prepared with TriZOL (GIBCO-BRL, San Diego, California, United States). cDNA was generated from the RNA using SuperRT (HT Biotechnology Ltd, Cambridge, England) and an oligo(dT) linker [SKT: GCTCTAGAAC TAGTGGATCCC(T) $\left.)_{20}\right]$. To analyze trt-1 transcription, PCR with primers DY15 (GCACCAACGATTAAGAGTTCAC) and DY16 (TTATTGCAACTTTATCAAAAATTGAC) was performed using cDNA obtained from N2 and mutant strains as a template. The spliced leader of trt-1 mRNA was determined by PCR of N2 cDNA using either an SL1 (GGTTTAATTACCCAAGTTTGAG) or SL2 (GGTTTTAACC CAGTTACTCAAG) oligo as a forward primer and DY16.

Rescue and complementation. N2 genomic DNA, pCes1943 (gift from Diana Janke), and pSA1 (carrying the trt-1 locus) were digested with PvuII, NruI, and ScaI, respectively. Mixtures of $100 \mathrm{ng}$ of N2 genomic DNA, 2 ng of pCes1943, and $1 \mathrm{ng}$ of pSA1 were injected into trt-1(e2727) and trt-1(ok410). Rol and non-Rol siblings were propagated in parallel and scored for sterility. For noncomplementation analysis, $e 2727$, ypl, or tm899 was placed in trans to unc-29 or trt-1(ok410),unc-29, and trans-heterozygotes were propagated by singling six non-Unc L4s each generation and selecting for the segregation of unc-29.

Analysis of mutant phenotypes at sterility. Fifty L4 larvae of each genotype were singled on NGM plates and scored for progeny after $48 \mathrm{~h}$. Worms that produced progeny were passaged using one L 4 per

\section{References}

1. Nakamura TM, Morin GB, Chapman KB, Weinrich SL, Andrews WH, et al. (1997) Telomerase catalytic subunit homologs from fission yeast and human. Science 277: 955-959.

2. Meyerson M, Counter CM, Eaton EN, Ellisen LW, Steiner P, et al. (1997) hEST2, the putative human telomerase catalytic subunit gene, is upregulated in tumor cells and during immortalization. Cell 90: 785-795.

3. Greider CW, Blackburn EH (1989) A telomeric sequence in the RNA of parent until a line became sterile (no progeny within $48 \mathrm{~h}$ post-L4). Sterile animals were stained with DAPI and scored for germline size, the presence of sperm and oocytes, the number of intestinal bridges, and chromosome number in oocytes.

Life span analysis. $\operatorname{trt}-1(0 k 410)$ strains were outcrossed numerous times to rejuvenate telomere length, and homozygous F2 lines were grown to sterility at $20^{\circ} \mathrm{C}$. Given that a variety of factors can affect $C$. elegans life span, it is best to perform analysis of different strains concurrently, under identical experimental conditions. For example, note the variation in maximum life span for the independent $o k 410$ and $\operatorname{tm} 899$ experiments (Figures 6 and S3). Therefore, frozen stocks of trt-1 mutants were created every four generations and then thawed and passaged on NGM plates for two generations prior to life span analysis. Each experiment was initiated with 100 L4 stage worms per genotype (six per plate). Animals were transferred every day for 1 week to separate them from their progeny and thereafter once a week. Worm viability was scored every day, and death was recorded when they failed to respond to light touch with a platinum pick. Worms that crawled off the plate and worms that died from an Egg Laying-Defective phenotype were excluded from the analysis. $P$ values were determined with the log-rank (Mantel-Cox) test using SAS software. Note that a recent study of telomere length and life span in C. elegans also relied on frozen stocks to examine life span for strains that were passaged for varying numbers of generations (Junho Lee, personal communication) [57].

\section{Supporting Information}

Figure S1. Telomere Shortening Is Observed for All Four trt-1 Alleles Found at DOI: 10.1371/journal.pgen.0020018.sg001 (3.0 MB TIF).

Figure S2. Additional TERT Multiple Sequence Alignments Found at DOI: 10.1371/journal.pgen.0020018.sg002 (1.9 MB TIF).

Figure S3. Life Span Is Normal for Both Early and Late Generations of Independent trt-1(ok410) and trt-1(tm899) Strains Found at DOI: 10.1371/journal.pgen.0020018.sg003 (1.6 MB TIF).

\section{Accession Numbers}

The GenBank (http://www.ncbi.nlm.nih.gov/Genbank/index.html) accession numbers for the proteins shown in Figure 2 are CeTRT-1 (NP_492374), CbTRT-1 (CAE66970), CrTRT-1 (DQ178631), hTERT (AAG23289), fTERT (AAX59693), Est2p (AAB64520), TtTERT (AAC39140), and the Brugia malayi T-motif clone (AI658475).

\section{Acknowledgments}

We thank the C. elegans Gene Knockout Consortium and the National Bioresource Project for the Experimental Animal C. elegans (Shohei Mitani) for providing the deletions trt-1(ok410) and trt-1(tm899), respectively; the C. elegans Genetics Center for providing strains; A. Coulson for plasmids; members of the Ahmed lab for discussion, technical assistance, and critical reading of the manuscript; and members of the Goldstein lab for discussion.

Author contributions. BM, IC, ML, JH, and SA conceived and designed the experiments. BM, IC, YL, ML, and SA performed the experiments and analyzed the data. AG provided materials, lab space, and help with experiments. BM, IC, ML, JH, and SA wrote the paper.

Funding. IC is the recipient of a University of North Carolina Lineberger Comprehensive Cancer Center postdoctoral fellowship. BM, YL, ML, and SA were supported by National Institutes of Health grant GM066228. This work was supported in part by CR-UK CDA (AG) and by the UK Medical Research Council.

Competing interests. The authors have declared that no competing interests exist.

Tetrahymena telomerase required for telomere repeat synthesis. Nature 337 : 331-337.

4. Lingner J, Cooper JP, Cech TR (1995) Telomerase and DNA end replication: No longer a lagging strand problem? Science 269: 1533-1534.

5. Harley CB, Futcher AB, Greider CW (1990) Telomeres shorten during ageing of human fibroblasts. Nature 345: 458-460.

6. Bodnar AG, Ouellette M, Frolkis M, Holt SE, Chiu CP, et al. (1998) Extension of life-span by introduction of telomerase into normal human cells. Science 279: 349-352. 
7. Wyllie FS, Jones CJ, Skinner JW, Haughton MF, Wallis C, et al. (2000) Telomerase prevents the accelerated cell ageing of Werner syndrome fibroblasts. Nat Genet 24: 16-17.

8. Rudolph KL, Chang S, Lee HW, Blasco M, Gottlieb GJ, et al. (1999) Longevity, stress response, and cancer in aging telomerase-deficient mice. Cell 96: 701-712.

9. Du X, Shen J, Kugan N, Furth EE, Lombard DB, et al. (2004) Telomere shortening exposes functions for the mouse Werner and Bloom syndrome genes. Mol Cell Biol 24: 8437-8446.

10. Chang S, Multani AS, Cabrera NG, Naylor ML, Laud P, et al. (2004) Essential role of limiting telomeres in the pathogenesis of Werner syndrome. Nat Genet 36: 877-882.

11. Wicky C, Villeneuve AM, Lauper N, Codourey L, Tobler H, et al. (1996) Telomeric repeats (TTAGGC)n are sufficient for chromosome capping function in Caenorhabditis elegans. Proc Natl Acad Sci U S A 93: 8983-8988.

12. Ahmed S, Hodgkin J (2000) MRT-2 checkpoint protein is required for germline immortality and telomere replication in C. elegans. Nature 403: $159-164$.

13. Boulton SJ, Gartner A, Reboul J, Vaglio P, Dyson N, et al. (2002) Combined functional genomic maps of the C. elegans DNA damage response. Science 295: $127-131$

14. Hofmann ER, Milstein S, Boulton SJ, Ye M, Hofmann JJ, et al. (2002) Caenorhabditis elegans HUS-1 is a DNA damage checkpoint protein required for genome stability and EGL-1-mediated apoptosis. Curr Biol 12: 1908-1918.

15. Page MF, Carr B, Anders KR, Grimson A, Anderson P (1999) SMG-2 is a phosphorylated protein required for mRNA surveillance in Caenorhabditis elegans and related to Upf1p of yeast. Mol Cell Biol 19: 5943-5951.

16. Malik HS, Burke WD, Eickbush TH (2000) Putative telomerase catalytic subunits from Giardia lamblia and Caenorhabditis elegans. Gene 251: 101-108.

17. Blumenthal T, Evans D, Link CD, Guffanti A, Lawson D, et al. (2002) A global analysis of Caenorhabditis elegans operons. Nature 417: 851-854.

18. Xia J, Peng Y, Mian IS, Lue NF (2000) Identification of functionally important domains in the $\mathrm{N}$-terminal region of telomerase reverse transcriptase. Mol Cell Biol 20: 5196-5207.

19. Moriarty TJ, Huard S, Dupuis S, Autexier C (2002) Functional multimerization of human telomerase requires an RNA interaction domain in the $\mathrm{N}$ terminus of the catalytic subunit. Mol Cell Biol 22: 1253-1265.

20. Armbruster BN, Banik SS, Guo C, Smith AC, Counter CM (2001) Nterminal domains of the human telomerase catalytic subunit required for enzyme activity in vivo. Mol Cell Biol 21: 7775-7786.

21. Friedman KL, Cech TR (1999) Essential functions of amino-terminal domains in the yeast telomerase catalytic subunit revealed by selection for viable mutants. Genes Dev 13: 2863-2874.

22. Lai CK, Mitchell JR, Collins K (2001) RNA binding domain of telomerase reverse transcriptase. Mol Cell Biol 21: 990-1000.

23. Beattie TL, Zhou W, Robinson MO, Harrington L (2001) Functional multimerization of the human telomerase reverse transcriptase. Mol Cell Biol 21: 6151-6160.

24. Banik SS, Guo C, Smith AC, Margolis SS, Richardson DA, et al. (2002) Cterminal regions of the human telomerase catalytic subunit essential for in vivo enzyme activity. Mol Cell Biol 22: 6234-6246.

25. Huard S, Autexier C (2004) Human telomerase catalyzes nucleolytic primer cleavage. Nucleic Acids Res 32: 2171-2180.

26. Porter SE, Greenwell PW, Ritchie KB, Petes TD (1996) The DNA-binding protein Hdflp (a putative $\mathrm{Ku}$ homologue) is required for maintaining normal telomere length in Saccharomyces cerevisiae. Nucleic Acids Res 24 $582-585$.

27. Nugent CI, Bosco G, Ross LO, Evans SK, Salinger AP, et al. (1998) Telomere maintenance is dependent on activities required for end repair of doublestrand breaks. Curr Biol 8: 657-660.

28. Boulton SJ, Jackson SP (1998) Components of the Ku-dependent nonhomologous end-joining pathway are involved in telomeric length maintenance and telomeric silencing. EMBO J 17: 1819-1828.

29. Baumann P, Cech TR (2000) Protection of telomeres by the Ku protein in fission yeast. Mol Biol Cell 11: 3265-3275.

30. Riha K, Shippen DE (2003) Ku is required for telomeric C-rich strand maintenance but not for end-to-end chromosome fusions in Arabidopsis. Proc Natl Acad Sci U S A 100: 611-615.

31. Gravel S, Larrivee M, Labrecque P, Wellinger RJ (1998) Yeast Ku as a regulator of chromosomal DNA end structure. Science 280: 741-744.

32. Kironmai KM, Muniyappa K (1997) Alteration of telomeric sequences and senescence caused by mutations in RAD50 of Saccharomyces cerevisiae. Genes Cells 2: 443-455.

33. Gartner A, Milstein S, Ahmed S, Hodgkin J, Hengartner MO (2000) A conserved checkpoint pathway mediates DNA damage-induced apoptosis and cell cycle arrest in C. elegans. Mol Cell 5: 435-443.

34. Lundblad V, Blackburn EH (1993) An alternative pathway for yeast telomere maintenance rescues est1- senescence. Cell 73: 347-360.
35. Bryan TM, Marusic L, Bacchetti S, Namba M, Reddel RR (1997) The telomere lengthening mechanism in telomerase-negative immortal human cells does not involve the telomerase RNA subunit. Hum Mol Genet 6: $921-$ 926.

36. Hemann MT, Rudolph KL, Strong MA, DePinho RA, Chin L, et al. (2001) Telomere dysfunction triggers developmentally regulated germ cell apoptosis. Mol Biol Cell 12: 2023-2030.

37. Karlseder J, Broccoli D, Dai Y, Hardy S, de Lange T (1999) p53- and ATMdependent apoptosis induced by telomeres lacking TRF2. Science 283: 1321-1325.

38. Gartner A, MacQueen AJ, Villeneuve AM (2004) Methods for analyzing checkpoint responses in Caenorhabditis elegans. Methods Mol Biol 280: 257274.

39. Alpi A, Pasierbek P, Gartner A, Loidl J (2003) Genetic and cytological characterization of the recombination protein RAD-51 in Caenorhabditis elegans. Chromosoma 112: 6-16.

40. Rinaldo C, Bazzicalupo P, Ederle S, Hilliard M, La Volpe A (2002) Roles for Caenorhabditis elegans rad-51 in meiosis and in resistance to ionizing radiation during development. Genetics 160: 471-479.

41. Kitagawa R, Rose AM (1999) Components of the spindle-assembly checkpoint are essential in Caenorhabditis elegans. Nat Cell Biol 1: 514-521.

42. Howe M, McDonald KL, Albertson DG, Meyer BJ (2001) HIM-10 is required for kinetochore structure and function on Caenorhabditis elegans holocentric chromosomes. J Cell Biol 153: 1227-1238.

43. Bryan TM, Goodrich KJ, Cech TR (2000) Telomerase RNA bound by protein motifs specific to telomerase reverse transcriptase. Mol Cell 6: 493499.

44. Chen JL, Greider CW (2004) An emerging consensus for telomerase RNA structure. Proc Natl Acad Sci U S A 101: 14683-14684.

45. The C. elegans Sequencing Consortium (1998) Genome sequence of the nematode C. elegans: A platform for investigating biology. Science 282: 2012-2018.

46. Stein LD, Bao Z, Blasiar D, Blumenthal T, Brent MR, et al. (2003) The genome sequence of Caenorhabditis briggsae: A platform for comparative genomics. PLoS Biol 1: e45. DOI: 10.1371/journal.pbio.0000045

47. Naito T, Matsuura A, Ishikawa F (1998) Circular chromosome formation in a fission yeast mutant defective in two ATM homologues. Nat Genet 20: 203-206.

48. Ritchie KB, Mallory JC, Petes TD (1999) Interactions of TLC1 (which encodes the RNA subunit of telomerase), TEL1, and MEC1 in regulating telomere length in the yeast Saccharomyces cerevisiae. Mol Cell Biol 19: 6065-6075.

49. Chan SW, Chang J, Prescott J, Blackburn EH (2001) Altering telomere structure allows telomerase to act in yeast lacking ATM kinases. Curr Biol 11: $1240-1250$.

50. Dahlen M, Olsson T, Kanter-Smoler G, Ramne A, Sunnerhagen P (1998) Regulation of telomere length by checkpoint genes in Schizosaccharomyces pombe. Mol Biol Cell 9: 611-621.

51. Longhese MP, Paciotti V, Neecke H, Lucchini G (2000) Checkpoint proteins influence telomeric silencing and length maintenance in budding yeast. Genetics 155: 1577-1591.

52. Weiss RS, Enoch T, Leder P (2000) Inactivation of mouse Hus1 results in genomic instability and impaired responses to genotoxic stress. Genes Dev 14: $1886-1898$

53. Parrilla-Castellar ER, Arlander SJ, Karnitz L (2004) Dial 9-1-1 for DNA damage: The Rad9-Hus1-Rad1 (9-1-1) clamp complex. DNA Repair (Amst) 3: 1009-1014.

54. Albertson DG, Thomson JN (1993) Segregation of holocentric chromosomes at meiosis in the nematode, Caenorhabditis elegans. Chromosome Res 1: $15-26$.

55. Hillers KJ, Villeneuve AM (2003) Chromosome-wide control of meiotic crossing over in C. elegans. Curr Biol 13: 1641-1647.

56. Sarin KY, Cheung P, Gilison D, Lee E, Tennen RI, et al. (2005) Conditional telomerase induction causes proliferation of hair follicle stem cells. Nature 436: $1048-1052$

57. Joeng KS, Song EJ, Lee KJ, Lee J (2004) Long lifespan in worms with long telomeric DNA. Nat Genet 36: 607-611.

58. Raices M, Maruyama H, Dillion A, Karlseder J (2006) Uncoupling of longevity and telomere length in C. elegans. PLoS Genet 1: e30. DOI: 10. 1371/journal.pgen.0010030.

59. Hahn WC (2003) Role of telomeres and telomerase in the pathogenesis of human cancer. J Clin Oncol 21: 2034-2043.

60. Sulston J, Hodgkin J (1988) Methods. In: Wood WB, editor. The nematode Caenorhabditis elegans. Plainview (New York): Cold Spring Harbor Laboratory Press. pp. 587-606.

61. Hemann MT, Strong MA, Hao LY, Greider CW (2001) The shortest telomere, not average telomere length, is critical for cell viability and chromosome stability. Cell 107: 67-77. 